\title{
Noninvasive Measurement of Intracranial Pressure: Is It Possible?
}

\author{
Tomasz Czarnik, MD, Ryszard Gawda, MD, Dariusz Latka, MD, PhD, Waldemar Kolodziej, MD, \\ Katarzyna Sznajd-Weron, PhD, and Rafal Weron, PhD
}

Background: Some publications suggest a strong correlation between the intracranial pressure and the intraocular pressure. Other studies claim no correlation between these two physiologic variables. Our aim was to study whether the tonometry could be a useful method to evaluate intracranial pressure in patients with suspected intracranial abnormality.

Methods: We evaluated the correlation between the intracranial pressure and the intraocular pressure, the intracranial pressure and the mean arterial pressure, and the intraocular pressure and the mean arterial pressure in 22 patients, initially comatose, who were admitted to our hospital. All patients required the intracranial pressure monitoring on clinical grounds. Simultaneous measurements were performed and recorded.

Results: We calculated both the linear correlation coefficient and the Spearman rank-order correlation coefficient. We found significant correlation between the intraocular pressure and the mean arterial pressure in 12 patients; however, significant correlation between the intraocular pressure and the intracranial pressure was found in only 2 patients.

Conclusion: Tonometry is not an appropriate method for the assessment of intracranial pressure increases.

Key Words: Intracranial pressure, Intraocular pressure, Correlation, Tonometry, Invasive measurement.

J Trauma. 2007;62:207-211.
A ccording to the Brain Trauma Foundation, traumatic brain injury (TBI) is one of the major causes of disability, death, and economic cost to a modern society. ${ }^{1}$ The neurologic deterioration after TBI is the direct effect of the primary injury (at the moment of impact) and secondary injuries during prehospital period and in the emergency department (delayed insults to the brain). It is obvious that the moment of impact is an independent factor from emergency medical service efforts. But what is done during the prehospital and initial hospital period is crucial to the patient's outcome.

Maintaining an adequate cerebral perfusion pressure (CPP) is the mainstay of therapy for the TBI patient. ${ }^{2-4} \mathrm{We}$ can evaluate $\mathrm{CPP}$ by monitoring the intracranial pressure (ICP) and the systemic blood pressure (continuously and invasively). Indications for ICP monitoring are known and transparent (Glasgow Coma Score 3 to 8 with abnormal computed tomography [CT] scan or two or more of the following adverse features: age $>40$ years, motor posturing, systolic blood pressure $[\mathrm{SBP}]<90 \mathrm{~mm} \mathrm{Hg}) .{ }^{1}$ The intracranial pressure sensor is implanted in the emergency department (ED) or the intensive care unit (ICU) after initial admission CT scan.

Submitted for publication May 28, 2005.

Accepted for publication February 16, 2006.

Copyright () 2007 by Lippincott Williams \& Wilkins, Inc.

From the Department of Anesthesia and Critical Care, Regional Medical Center, Opole, Poland.

Address for reprints: Tomasz Czarnik, MD, Regional Medical Center, Opole, Poland; email: antocz@mp.pl.

DOI: 10.1097/01.ta.0000219128.29515.d5
Noninvasive methods of ICP measurement useful in the ICU such as transcranial Doppler (TCD), visual-evoked responses (VERs), brain stem auditory-evoked responses (BAERs), ophthalmodynamometry, scalp blood flow (SBF) measured by Laser Doppler, and impedance audiometry are known but still experimental. ${ }^{5-7}$ As far as our own experience is concerned, we wondered whether there was a correlation between ICP and the intraocular pressure (IOP), which would help to evaluate the CPP in the management of the critically ill patient.

Recent publications suggest a strong correlation between IOP and ICP. ${ }^{8}$ But the physiologic mechanism responsible for elevations of IOP remains unclear. Salman ${ }^{9}$ suggests that the cerebrospinal fluid (CSF) surrounding the optic nerve sheath transmits elevations of ICP through the eyeball, raising the IOP level. Other potential mechanisms suggest that the rise in the ophthalmic venous pressure (as the result of ICP elevation) could be transmitted directly to the ocular fluid raising $\mathrm{IOP}^{10}$ or increased venous pressure in the cavernous sinus (also the direct effect of ICP rise) is transmitted to episcleral veins by the superior ophthalmic vein and becomes the cause of IOP increase. ${ }^{11,12}$ The results of another study ${ }^{13}$ showed a linear correlation between the central retinal vein pressure and ICP. The venous outflow pressure of the central retinal vein was measured. This pressure (according to the study) reflects ICP because the central retinal vein passes through the optic nerve and then drains into the cavernous sinus and is independent from IOP. The method of measuring the central retinal vein pressure is called the ophthalmodynamometry. ${ }^{14}$

There are also publications claiming no correlation between IOP and ICP. Hayreh ${ }^{15}$ indicates that only an acute ICP increase of more than $50 \mathrm{~mm} \mathrm{Hg}$ may cause significant 
increase of IOP. However, such enormous ICP increases are clinically rare phenomena and there is no anatomic and physiologic evidence for the statement that raised ICP is directly transmitted through the eyeball raising the IOP. The subarachnoid space around the optic nerve is separated from the eyeball by a fibrous tissue, which prevents the ICP transmission to the eye. According to researchers, a rapid rise in ICP to $50 \mathrm{~mm} \mathrm{Hg}$ caused the increase in the ophthalmic venous pressure but had no significant effect on IOP. As far as the respiratory acidosis and alkalosis and prostaglandins (E1, E2, A) infusion that decreases both ICP and IOP is concerned, researchers claim that these changes represent independent physiologic roots and could not be regarded as evidence for a correlation between ICP and IOP.

\section{PATIENTS AND METHODS}

We investigated the correlation not only between IOP and ICP, but also between IOP and the mean arterial pressure (MAP), and ICP and MAP. We tried to evaluate if a rapid, noninvasive assessment of ICP was possible in a patient with suspected intracranial abnormality (during prehospital period, in ED or ICU) during a period when the intraparenchymal ICP sensor had not yet been implanted.

The study included invasive continuous monitoring of ICP using the intraparenchymal sensor, invasive continuous monitoring of arterial blood pressure, and measuring IOP using the Schioetz Tonometer. IOP measurement was conducted in 8-hour intervals and also when ICP had changed by $5 \mathrm{~mm} \mathrm{Hg}$ or more. All IOP measurements were performed by the same person (Tomasz Czarnik) with the use of the same equipment. We investigated patients for whom ICP measurement was necessary on clinical grounds. We had the approval from the local ethics committee. Patients with a significant facial and ocular trauma, a history of glaucoma, and corneal disease were excluded.

The method of ICP monitoring included intraparenchymal placement of the intracranial pressure sensor (The MicroSensor-Codman, Johnson \& Johnson Professional, Randolph, MA). The procedure usually took place in the operating room on the day of admission. The transducer was calibrated before insertion and then the sensor was advanced 3 to $4 \mathrm{~cm}$ into the brain parenchyma. The ICP pressure value of $20 \mathrm{~mm} \mathrm{Hg}$ or less was regarded as normal. After 5 to 7 days of measurement, the intracranial pressure sensor was removed. ${ }^{16-23}$ Continuous invasive measurement of blood pressure was performed during ICP monitoring using standard bedside transducers leveled to the heart. ${ }^{24}$

The IOP was measured by using the Schioetz Eye Tonometer (Rudolf Riester $\mathrm{GmbH}$ ) in both eyes and a mean IOP was calculated. Immediately before the IOP measurement was taken, the tonometer was cleaned and then placed on the test block (testing procedure). If the pointer had been set to zero, the start of measurement was possible. The patient was in a recumbent position. The patient was sedated if it had

\begin{tabular}{ccc}
\hline Table 1 Study Patients $(\mathbf{N}=\mathbf{2 2})$ & & \\
\hline Intracranial Abnormality & $\mathrm{n}$ & Percent \\
\hline Traumatic brain injury & 12 & 55 \\
Subarachnoid hemorrhage & 4 & 18 \\
Intracerebral hemorrhage & 6 & 27 \\
\hline
\end{tabular}

been necessary for proper measurement and patient safety (to stop intracranial and hemodynamic response). The midazolam was used for sedation in a dose of 2 to $5 \mathrm{mg}(0.05 \mathrm{mg} / \mathrm{kg}$ body weight) intravenously. After anesthetizing the cornea with a local anesthetic (Alcaine-Proxymetacaini hydrochloridum, Alcon-Couvreur), the tonometer was placed in a vertical position at the center of the cornea. Reliable pressure values were recorded when the pointer had shown a pulse. According to the manufacturer, the pressure of a healthy eye was approximately $16 \mathrm{~mm} \mathrm{Hg}$ (average value).

The MAP, ICP, and IOP values were recorded in the measurement protocol at the same time. The approach to the therapy of patients followed general critical care principles and was CPP directed. All patients were initially mechanically ventilated (normoventilated) without positive end-expiratory pressure (PEEP) institution. $^{25}$ The management of elevated ICP included 15-degree head-up position, osmotherapy, mechanical normoventilation, avoidance of jugular venous obstruction, normovolemia, prevention of hyperthermia and hyperglycemia, moderate cooling, surgical intervention, and barbiturate coma as the last resort. The treatment for ICP was usually initiated at an upper threshold of $20 \mathrm{~mm} \mathrm{Hg}$. According to CPP, the aim was to maintain it above $60 \mathrm{~mm} \mathrm{Hg}$ (above lower limit of autoregulation).

Twenty-two patients were joined in the study, 18 men and 4 women. There were no children in this group. In all, 287 measurements were performed (6-19 per patient). Sixteen patients had elevated ICP (above $20 \mathrm{~mm} \mathrm{Hg}$ ): the lowest ICP was $5 \mathrm{~mm} \mathrm{Hg}$ and the highest was $110 \mathrm{~mm} \mathrm{Hg}$. Thirteen patients had elevated IOP (above $16 \mathrm{~mm} \mathrm{Hg}$ ): the lowest IOP was $4.2 \mathrm{~mm} \mathrm{Hg}$ and the highest was $46.9 \mathrm{~mm} \mathrm{Hg}$. There were TBI patients, subarachnoid hemorrhage (SAH) patients, and intracerebral hemorrhage $(\mathrm{ICH})$ patients not associated with TBI (Table 1). In the TBI group, such intracranial pathological abnormalities as cerebral contusion, intracranial hematomas, and diffuse axonal injury were revealed. All patients were comatose (Glasgow Coma Score 3-8) at admission. Outcome scales were not taken into account. The study was conducted between December 2003 and November 2004.

\section{Biostatistical Analysis}

We calculated both the linear correlation coefficient and the Spearman rank-order correlation coefficient. ${ }^{26} \mathrm{We}$ assumed that the dependence is significant if $p$ value $<0.05$ for both methods simultaneously. All statistical analyses and plots were done using Matlab 6.0, release 12 (The MathWorks). We measured the correlation of ICP to IOP, MAP to IOP, and MAP to ICP. 

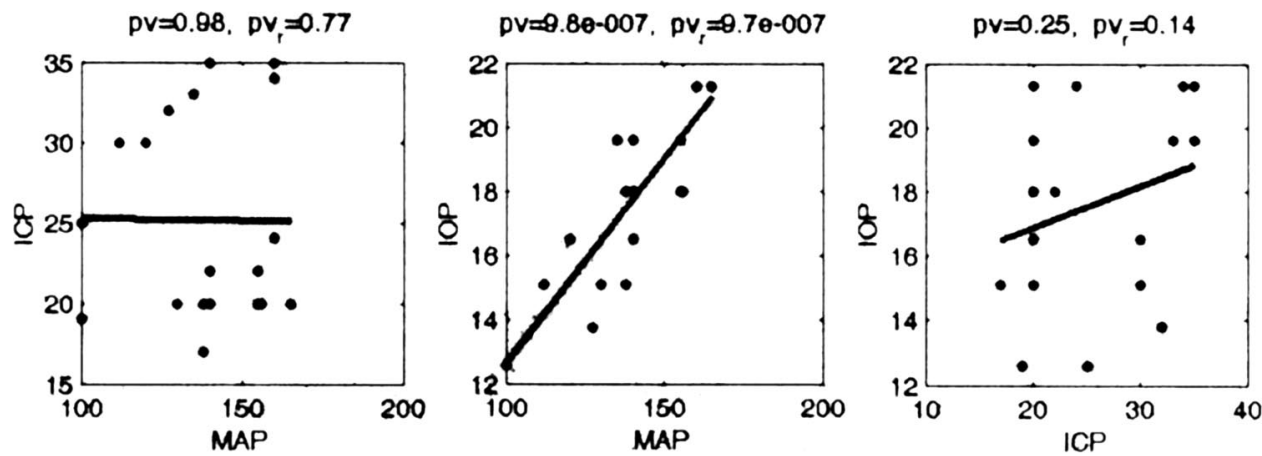

Fig. 1. Correlation plot between MAP and ICP, MAP and IOP, and ICP and IOP in patient 7. There is a significant correlation between $M A P$ and IOP (linear correlation coefficient $=0.8744 ; \mathrm{p}<0.0001$ ).

\section{RESULTS}

Out of the 22 patients, 5 patients had a positive linear correlation and 2 patients had a negative correlation of ICP to IOP. The range for the positive correlation coefficient was 0.5457 to 0.8762 , whereas for the negative it was -0.8252 to -0.6566 . However, calculating both linear and Spearman rank-order correlation coefficients, we found that the correlation between ICP and IOP was significant in only two patients (patients 14 and 22). Dependence analysis for a sample patient (patient 7) is presented in Figure 1. Dependence between ICP and IOP for all patients is presented in Figure 2. Note that patients with significant correlation be- tween ICP and IOP (patients 14 and 22) could be treated as special cases. The IOP values of patient 14 are extremely high compared with the corresponding values in the whole population. In spite of this, there were no special features in patients who had a positive correlation between ICP and IOP that could have lead to any conclusions.

We found a significant correlation between MAP and IOP in 12 patients (i.e., for $\sim 55 \%$ of the tested population). All of these patients have a positive correlation, which ranges from 0.5202 to 0.9650 for the linear correlation coefficient and is between 0.6520 and 0.9908 for the Spearman rankorder correlation coefficient. However, we found that the

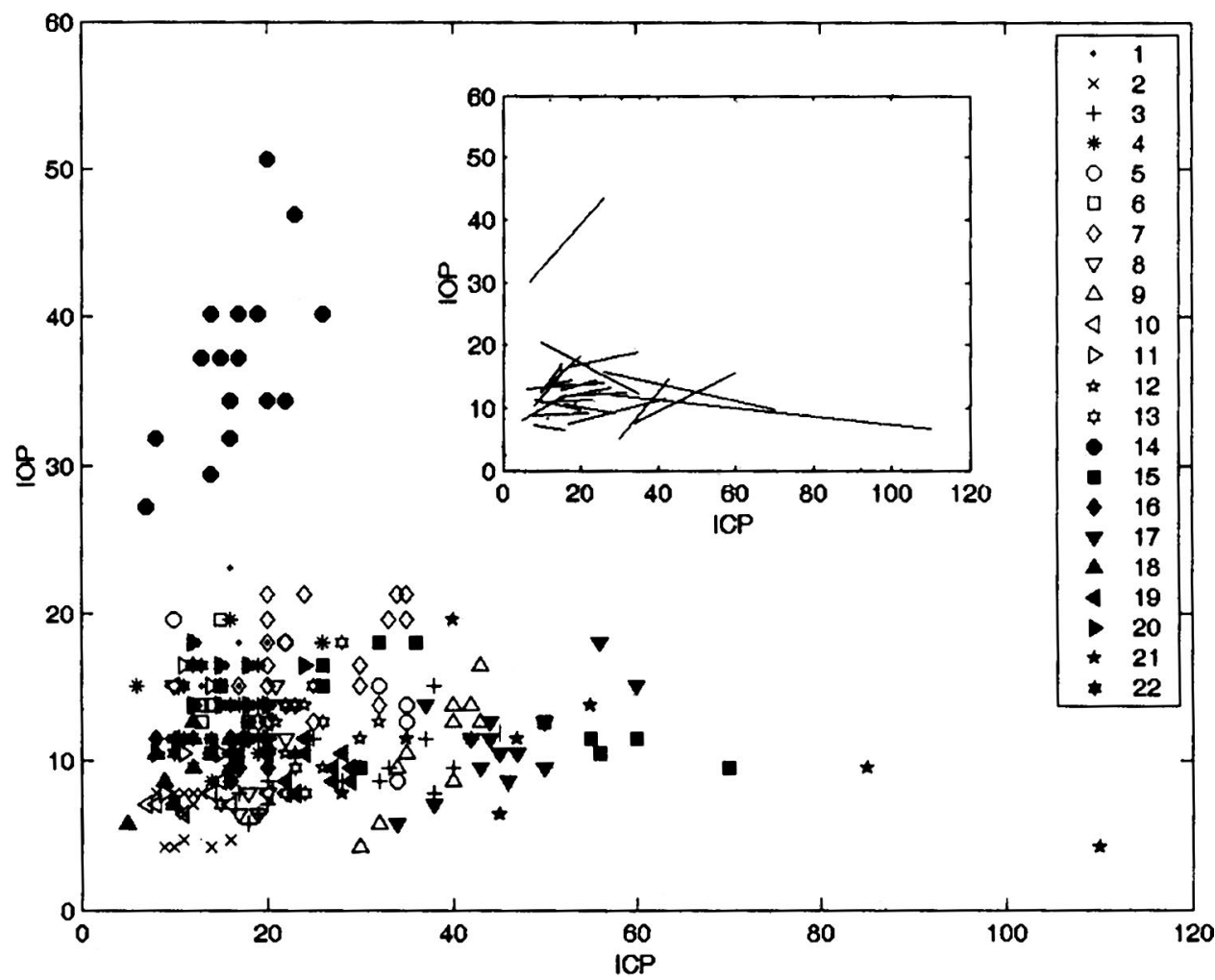

Fig. 2. Correlation plot between ICP and IOP for all patients. Each patient is denoted by a different symbol. Regression lines for each of the patients are depicted in the inset. Globally, no dependence can be observed for these data. Note the outlying observations related to patients 14 and 21 . 
correlation between MAP and ICP is significant in only three patients (patients 8, 9, and 10). The correlation between MAP and IOP is significant, but neither ICP and IOP nor MAP and ICP are correlated. Globally, linear dependence can be observed only between MAP and IOP.

\section{DISCUSSION}

The study suggests no correlation between IOP and ICP and significant correlation between MAP and IOP. It contributes to the controversy that surrounds the recent literature. Recent articles by Sheeran et al. ${ }^{8}$ and Lashutka et al. ${ }^{11}$ support the use of intraocular pressure as an acute measure of intracranial pressure. Our study utilizes a slightly different approach that might be the cause of negative result. Each patient was observed for a longer period of time (2 to 6 days). Minimal number of measurements per day was three. Hemodynamic instability was not a cause of exclusion. There were no dramatic changes in central venous pressure but frequent changes in arterial pressure. The MAP fluctuations were associated with significant IOP fluctuations in 12 patients (positive correlation) without marked changes in ICP. It could suggest that MAP-related regulation of IOP is probably present in patients with intracranial abnormalities.

Results from animal studies suggest that large ICP changes are necessary to cause IOP rise. ${ }^{15} \mathrm{We}$ observed large rises in ICP above the level of $50 \mathrm{~mm} \mathrm{Hg}$ in three patients $(15,17$, and 21$)$. In patient 15 , the IOP did not change; in patient 17 , the IOP rose significantly but the MAP was very high; in patient 21, the ICP value reached $110 \mathrm{~mm} \mathrm{Hg}$ and the IOP dropped to $4.2 \mathrm{~mm} \mathrm{Hg}$ and it was associated with brain death. It could suggest that the intraocular pressure is not associated directly with the intracranial pressure but rather with cerebral hemodynamics (cerebral blood flow).

Physiologic and anatomic relationships between ICP and IOP are still unknown and potential ways of influence are hypothetical. The cerebrospinal fluid is separated from the eyeball and the optic nerve by a fibrous tissue that limits pressure transmissions. The hypothesis that elevations of ICP could be transmitted via the cavernous sinus and the superior ophthalmic vein was not clinically verified. It could be explained by the fact that the superior ophthalmic vein is connected not only with the cavernous sinus but also with the facial vein (via the angular vein). Pressure elevations in the cavernous sinus theoretically might divert blood flow toward the facial vein via the superior ophthalmic vein preventing IOP rise (there are no valves in the superior ophthalmic vein). Linear correlation between the central retinal vein pressure and ICP could also be anatomically explained. The central retinal vein directly enters the cavernous sinus and has not alternative anastomosis. The central retinal vein pressure elevation and macular edema reflect intracranial hypertension.

As far as sedation is concerned, Carter et al. ${ }^{27}$ claim that intravenous administration of midazolam in a dose of $1 \mathrm{mg}$ does not lower IOP, whereas Hirlinger et al. ${ }^{28}$ observed a reduction in intraocular pressure after administration of midazolam in a much higher dose of $0.15 \mathrm{mg} / \mathrm{kg}$ intravenously with fentanyl after induction of anesthesia. Cesati et al. ${ }^{29}$ investigated IOP after intravenous midazolam premedication $(0.05 \mathrm{mg} / \mathrm{kg}$ body weight) and anesthesia induction. No reduction in IOP was observed. Such a dose of midazolam $(0.05 \mathrm{mg} / \mathrm{kg}$ body weight $)$ was chosen to prevent influence on the IOP examination.

We can expect that tonometry will not be an appropriate method for the assessment of ICP increases, particularly in patients suffering from TBI who are hemodynamically unstable when admitted to the ED setting.

\section{ACKNOWLEDGMENTS}

We would like to thank Prof. Wojciech Gaszynski, MD, for his advice in designing this study and scientific support.

\section{REFERENCES}

1. Guidelines for the Management of Severe Traumatic Brain InjuryA joint project of the Brain Trauma Foundation, American Association of Neurological Surgeons, Joint Section on Neurotrauma and Critical Care. New York: Brain Trauma Foundation, 2000.

2. Robertson CS. Management of Cerebral Perfusion Pressure after Traumatic Brain Injury. Anesthesiology. 2001;95:1513-1517.

3. Jackson RGM, Sales KM, McLaughlin DP, Stamford JA. Traumatic brain injury: from model to man. Curr Anaesthesia Crit Care. 2002; 13:343-355.

4. Goh J, Gupta AK. The management of head injury and intracranial pressure. Curr Anaesthesia Crit Care. 2002;13:129-137.

5. Layon AJ, Gabrielli A. Elevated intracranial pressure. In: Textbook of Neurointensive Care. Layon JA, Gabrielli A, Friedmann WA, eds. Philadelphia: Saunders; 2004:709-732.

6. Bellner J, Romner B, Reinstrup P, et al. Transcranial Doppler sonography pulsatility index (PI) reflects intracranial pressure (ICP). Surg Neurol. 2004;62:45-51.

7. Paulat K, Brucher R, Russel D. Noninvasive monitoring of intracranial pressure and compliance. Ann Thorac Surg. 2002;73:371.

8. Sheeran P, Bland JM, Hall GM. Intraocular pressure changes and alterations in intracranial pressure (letter). Lancet. 2000;355:899.

9. Salman MS. Can intracranial pressure be measured non-invasively? Lancet. 1997;350:1367.

10. Hartmann A. Non-invasive measurement of intracranial pressure (letter). Lancet. 1998;351:524.

11. Lashutka MK, Chandra A, Murray HN, et al. The Relationship of Intraocular Pressure to Intracranial Pressure. Ann Emerg Med. 2004; 43:585-591.

12. Lehman RA, Krupin T, Podos SM. Experimental effect of intracranial hypertension upon intraocular pressure. $J$ Neurosurg. 1972;36:60-66.

13. Firsching R, Schutze M, Motschmann M, et al. Non-invasive measurement of intracranial pressure (letter). Lancet. 1998; 351:523-524.

14. Dickerman RD, Smith GH, Langham-Roof L, et al. Intra-ocular pressure changes during maximal isometric contraction; Does this reflect intra-cranial pressure or retinal venous pressure? Neurologic Res. 1999;21:243-246.

15. Hayreh SS. Non-invasive measurement of intracranial pressure (letter). Lancet. 1998;351:524-525.

16. Signorini DF, Shad A, Piper IR, et al. A clinical evaluation of the Codman MicroSensor for intracranial pressure monitoring.

Br J Neurosurg. 1998;12:223-227. 
17. Rebuck JA, Murry KR, Rhoney DH, et al. Infection related to intracranial pressure monitors in adults: analysis of risk factors and antibiotic prophylaxis. J Neurol Neurosurg Psychiatry. 2000;69: 381-384.

18. Czosnyka M, Smielewski P, Piechnik S, et al. Critical closing pressure in cerebrovascular circulation. $J$ Neurol Neurosurg Psychiatry. 1999;66:606-611.

19. Thees C, Scholz M, Schaller MDC, et al. Relationship between intracranial pressure and critical closing pressure in patients with neurotrauma. Anesthesiology. 2002;96:595-599.

20. Czosnyka M, Smielewski P, Kirkpatrick P, et al. Monitoring of cerebral autoregulation in head injured patients. Stroke. 1996; 27:1829-1834.

21. Gupta AK. Monitoring the Injured Brain in the Intensive Care Unit. J Postgrad Med. 2002;48:218-225.

22. Ravi R, Morgan RJ. Intracranial pressure monitoring. Curr Anaesthesia Crit Care. 2003;14:229-235.

23. Gupta AK, Azami J. Update of neuromonitoring. Curr Anaesthesia Crit Care. 2002;13:120-128.
24. Bessman ES. Invasive monitoring, pacing techniques and automated and implantable defibrillators. In: Emergency Medicine. A Comprehensive Study Guide. 6th Ed. Tintinalli JE, Kelen GD, Stapczynski JS, eds. New York: McGraw-Hill; 2004:132-138.

25. Teba L, Viti A, Banks DE, et al. Intraocular pressure during mechanical ventilation with different levels of positive endexpiratory pressure. Crit Care Med. 1993;21:867-870.

26. Mickey RM, Dunn OJ, Clark VA. Applied Statistics: Analysis of Variance and Regression. Hoboken, NJ: Wiley; 2004.

27. Carter K, Faberowski LK, Sherwood MB, et al. A randomized trial of the effect of midazolam on intraocular pressure. J Glaucoma. 1999;8:204-207.

28. Hirlinger WK, Wick C, Stodtmeister R. Comparative study of the behavior of intraocular pressure in anesthesia induction by diazepam and midazolam. Anasth Intensivther Notfallmed. 1986; $21: 324-326$

29. Casati A, Aldegheri G, Fanelli G, et al. Lightwand intubation does not reduce the increase in intraocular pressure associated with tracheal intubation. J Clin Anesth. 1999;11:216-219. 